\title{
Correction to: Human ancestry indentification under resource constraints -- what can one chromosome tell us about human biogeographical ancestry?
}

Tanjin T. Toma, Jeremy M. Dawson and Donald A. Adjeroh*

\section{Correction to: BMC Med Genomics}

https://doi.org/10.1186/s12920-018-0412-4

After publication of this supplement article [1], it was flagged that there is a typo in the article's title.

Please be advised that the correct title is "Human ancestry identification under resource constraints -- what can one chromosome tell us about human biogeographical ancestry?"

That is, "indentification" should read "identification".

Published online: 09 January 2019

\section{Reference}

1. Toma, et al. Human ancestry indentification under resource constraints -- what

can one chromosome tell us about human biogeographical ancestry? BMC

Med Genet. 2018;11(Suppl 5). https://doi.org/10.1186/s12920-018-0412-4.

* Correspondence: don@csee.wvu.edu

Lane Department of Computer Science and Electrical Engineering, West

Virginia University, Morgantown, WV, USA 